\title{
Reduced inflammatory cell recruitment and tissue damage in spinal cord injury by acellular spinal cord scaffold seeded with mesenchymal stem cells
}

\author{
YU-HAI WANG ${ }^{1 *}$, JIAN CHEN $^{2 *}$, JING ZHOU $^{3 *}$, FENG NONG $^{4}$, JIN-HAN LV $^{1}$ and JIA LIU ${ }^{4}$ \\ ${ }^{1}$ Department of Orthopedics, Ningxia People's Hospital, First Affiliated Hospital of Northwest University for Nationalities, \\ Yinchuan, Ningxia 750000; ${ }^{2}$ Department of Orthopedic Surgery, Chongqing Three Gorges Central Hospital, \\ Chongqing 404000; ${ }^{3}$ Department of Anatomy, Youjiang Medical College for Nationalities; ${ }^{4}$ Department of Orthopedics, \\ Affiliated Hospital of Youjiang Medical College for Nationalities, \\ Baise, Guangxi 533000, P.R. China
}

Received August 19, 2015; Accepted September 26, 2016

DOI: $10.3892 /$ etm.2016.3941

\begin{abstract}
Therapy using acellular spinal cord (ASC) scaffolds seeded with bone marrow stromal cells (BMSCs) has previously been shown to restore function of the damaged spinal cord and improve functional recovery in a rat model of acute hemisected spinal cord injury (SCI). The aim of the present study was to determine whether BMSCs and ASC scaffolds promote the functional recovery of the damaged spinal cord in a rat SCI model through regulation of apoptosis and immune responses. Whether this strategy regulates secondary inflammation, which is characterized by the infiltration of immune cells and inflammatory mediators to the lesion site, in SCI repair was investigated. Basso, Beattie, and Bresnahan scores revealed that treatment with BMSCs seeded into an ASC scaffold led to a significant improvement in motor function recovery compared with treatment with an ASC scaffold alone or untreated controls at 2 and 8 weeks after surgery $(\mathrm{P}<0.05)$. Two weeks after transplantation, the BMSCs seeded into an ASC scaffold significantly decreased the number of terminal deoxynucleotidyl transferase dUTP nick end labeling-positive cells, as compared with the ASC scaffold only and control groups. These results suggested that the use of BMSCs decreased the apoptosis of neural cells and thereby limited tissue damage at the lesion site. Notably, the use of BMSCs with an ASC scaffold also decreased the recruitment of macrophages (microglia; $\mathrm{P}<0.05)$ and $\mathrm{T}$ lymphocytes $(\mathrm{P}<0.05)$ around the SCI site, as indicated
\end{abstract}

Correspondence to: Dr Jia Liu, Department of Orthopedics, Affiliated Hospital of Youjiang Medical College for Nationalities, 18 Zhongshan II Road, Baise, Guangxi 533000, P.R. China E-mail: liujia0111@live.cn

*Contributed equally

Key words: spinal cord injury, inflammatory, mesenchymal stem cells, scaffold by immunofluorescent markers. By contrast, there was no inhibition of the inflammatory response in the control and ASC scaffold only groups. BMSCs regulated inflammatory cell recruitment to promote functional recovery. However, there was no significant difference in IgM-positive expression among the three groups $(\mathrm{P}>0.05)$. The results of this study demonstrated that BMSCs seeded into ASC scaffolds for repair of spinal cord hemisection defects promoted functional recovery through the early regulation of inflammatory cell recruitment with inhibition of apoptosis and secondary inflammation.

\section{Introduction}

The natural pathology of spinal cord injury (SCI) progression usually includes primary and secondary phases of injury that finally result in severe sensory and motor deficits beyond the level of the injury site (1). Primary traumatic mechanical SCI often results in the death of a number of neurons that can neither be recovered nor regenerated. The secondary SCI process is characterized by demyelination, neural apoptosis and post-traumatic inflammatory reactions as a homeostatic response aimed to clear debris at the lesion site, while simultaneously preserving organ function (2-4). However, if the post-traumatic inflammatory reaction is uncontrolled, it can cause the initial lesion to be enlarged by means of additional axonal damage, oligodendrocyte death and demyelination with concomitant increased loss of neurological function, which is a potentially avoidable event that can be regulated or reduced by avoiding additional neural death and defending against functional deficits $(5,6)$. There is evidence that leukocytes, particularly $\mathrm{T}$ lymphocytes and macrophages that infiltrate the injured spinal cord, are directly involved in the pathogenesis and extension of SCI (7).

SCI remains a very complex medical and psychological challenge. Notably, previous studies conducted by authors of the present study demonstrated that the use of an acellular spinal cord (ASC) scaffold seeded with bone marrow stromal cells (BMSCs) conveyed potential benefits to the repair of 
SCIs by protecting native tissues and promoting functional recovery in a rat model of SCI $(8,9)$. However, whether stem cells or scaffolds are able to ameliorate secondary and extended inflammation to the lesion site, thereby contributing to SCI repair, has yet to be explored.

The aim of the present study was to investigate whether the use of an ASC scaffold seeded with BMSCs was sufficient to restore the damaged spinal cord and improve functional recovery in a rat spinal cord hemisected model of SCI through regulation of apoptosis and the local inflammatory response.

\section{Materials and methods}

BMSC proliferation and preparation of the ASC scaffold. BMSCs were prepared from the femurs and tibias of 6-week-old Sprague-Dawley (SD) rats, as described in a previous study (8). Briefly, BMSCs were isolated from the marrow cavity and then filtered through a $70-\mu$ m nylon mesh to remove any remaining clumps of tissue. After centrifugation at $200 \times \mathrm{g}$ for $5 \mathrm{~min}$, the collected cells were placed in $75-\mathrm{cm}^{2}$ culture flasks and cultured in Dulbecco's modified Eagle's medium containing 20\% fetal bovine serum (Gibco; Thermo Fisher Scientific, Inc., Waltham, MA, USA) and $1 \%$ penicillin and streptomycin (Invitrogen; Thermo Fisher Scientific, Inc.). Following incubation for $72 \mathrm{~h}$, non-adherent cells were removed by washing with phosphate-buffered saline (PBS). The culture medium was replaced every 4 days. The cells after three passages were used for subsequent experiments.

The ASC scaffold was prepared as described previously (9). Briefly, the thoracic spinal cords were harvested from sacrificed adult SD rats and the ASC was chemically extracted. The thoracic spinal cord was treated with a series of detergents consisting of distilled water, Triton X-100 (Amresco LLC, Solon, OH, USA) and sodium deoxycholate solution (Amresco LLC). All samples were then freeze-dried for $24 \mathrm{~h}$ and sterilized by irradiation with cobalt-60 gamma rays ( $3 \mathrm{kGy}$ ) prior to usage.

Spinal cord hemisection model and graft transplantation. The study protocol was approved by the Ethics Committee for Animal Research of Ningxia People's Hospital (Yinchuan, China) and performed in accordance with international standards for animal welfare. All surgical procedures were performed under anesthesia produced by the intraperitoneal injection of $10 \%$ chloral hydrate $(0.4 \mathrm{ml} / 100 \mathrm{~g}$; Guangzhou Chemical Plant, Guangzhou, China). A total of 24 adult male SD rats (body weight, 200-250 g) were provided by Ningxia Medical University Animal Center, Yinchuan, China) and maintained in housing with a constant temperature $\left(25^{\circ} \mathrm{C}\right.$, $50 \pm 5^{\circ} \mathrm{C}$ humidity), a 12 -h light/dark cycle and free access to food and water. Rats underwent laminectomy at the T9-10 level to expose one thoracic spinal cord segment. A small incision was made in the right dorsal spinal cord and a 22-gauge needle made of ethylene tetrafluoroethylene was used to remove $2 \mathrm{~mm}$ of the right hemicord (9). The rats were then randomly divided into three experimental groups according to the type of graft: i) Animals that received implantation of an ASC scaffold with BMSCs $(n=8)$, ii) animals that received implantation of an ASC scaffold with no BMSCs $(n=8)$, and iii) animals for which the lesion cavity was left empty, as a control group $(n=8)$. After surgery, all rats received daily subcutaneous ampicillin (100 $\mathrm{mg} / \mathrm{kg}$ ) for 3 days to avoid infection, and their bladders were expressed manually twice per day until bladder control was regained.

Behavioral analysis. Motor function of the right hind limb was assessed in an open-field via the Basso, Beattie and Bresnahan (BBB) locomotor scoring system on a scale from 0 to 21 (10). Briefly, individual rats were placed on an open field at 2 and 8 weeks after transplantation and observed for $4 \mathrm{~min}$ by two observers blinded to the treatment.

Tissue processing. The animals were anesthetized at the end of each study using an overdose of chloral hydrate. After transcardial perfusion, which was performed as previously described (9), the spinal cords were dissected, fixed in $4 \%$ paraformaldehyde solution overnight, cryoprotected in a $30 \%$ sucrose solution in PBS, then embedded in Tissue-Tek Optimal Cutting Temperature compound mounting media (Sakura Finetek USA, Inc., Torrance, CA, USA) and cut into $5-\mu \mathrm{m}$ coronal sections using a cryostat. Every seventh section was stained with hematoxylin and eosin (H\&E).

Histological and quantitative analysis. Immunofluorescence was used to assess the presence of markers of inflammatory cells around the SCI lesion site at 2 weeks after transplantation and the sections were stained with $H \& E$ to observe morphological aspects of the spinal cord from rats in each experimental group ( $n=3$ per group) at 8 weeks after transplantation. Briefly, spinal cord sections were washed with PBS and permeabilized with $0.1 \%$ Triton X-100 for 5 min. After washing in PBS again, the sections were blocked with $10 \%$ bovine serum albumin (Sigma-Aldrich; Merck Millipore, Darmstadt, Germany). Fluorescein isothiocyanate-labeled primary antibodies against immunoglobulin M (FITC anti-rat IgM; dilution, 1:100; cat. no. RUO 400801; BioLegend, Inc., San Diego, CA, USA), macrophages (anti-CD68/FITC; $100 \mu \mathrm{g}$; dilution, 1:100; cat. no. BS-0649R; Bioss, Inc., Woburn, MA, USA), and T lymphocytes (anti-CD5/FITC; dilution, 1:100; cat. no. BS-10218R; Bioss, Inc.) were used to evaluate each section. Finally, each section was stained with 4,6-diamidino-2-phenylindole (DAPI; Molecular Probes; Thermo Fisher Scientific, Inc.) to visualize nucleated cells.

Terminal deoxynucleotidyl transferase dUTP nick end labeling (TUNEL). A TUNEL kit (Nanjing Keygen Biotech Co., Ltd., Nanjing, China) was used to evaluate the extent of apoptosis, according to the manufacturer's instructions. Tissue sections were analyzed ( $n=3$ per group) to detect the localization of apoptotic cells, indicated by green fluorescence, and cell nuclei, indicated by blue fluorescence using fluorescence microscopy.

Quantitation of positive cells in spinal cord tissues. Slides prepared as outlined above were examined using a light microscope at x40 magnification. Positive cells were counted in three representative sections from at least four animals in each group. The results are presented as the mean plus standard deviation number of positive cells per field. 
Statistical analysis. Quantitative data from locomotor behavior scores and the number of cells positive for markers of inflammation are expressed as the mean \pm standard deviation or mean plus standard deviation, respectively. $\mathrm{P}<0.05$ was considered to indicate a statistically significant difference. Statistical analysis was performed by analysis of variance followed by Bonferroni's post-hoc test (multiple-comparisons tests) among three groups using SPSS statistical software (version 13.0; SPSS, Inc., Chicago, IL, USA).

\section{Results}

Isolation of BMSCs and seeding of ASC scaffolds transplanted into hemisected spinal cords. BMSCs were successfully isolated from the marrow of femurs and tibias of anesthetized rats. After adherence and passage three times, the BMSCs were collected ex vivo via chemical extraction and seeded in ASC scaffolds. The ASC scaffolds with or without seeded BMSCs were implanted immediately into spinal cord hemisection cavities to bridge lesion sites after surgery.

Assessment of locomotor behavior. All animals survived surgery and were subsequently used for analysis. The BBB scoring behavioral assessment system was used to assess locomotor recovery. The rats in each experimental group showed gradual improvement in functional recovery during the convalescent period. However, at 2 and 8 weeks after SCI, the rats implanted with ASC scaffolds seeded with BMSCs showed significantly enhanced BBB scores compared with the ASC group and the control group (12.45 \pm 0.54 and 17.92 \pm 0.46 for the BMSCs + ASC group vs. 9.32 \pm 0.70 and 12.23 \pm 0.80 for the ASC group and $5.23 \pm 0.67$ and $7.93 \pm 0.46$ for the control group, respectively). These results showed that BMSCs seeded in ASC scaffolds improved the recovery of motor function following surgery (Fig. 1).

Histological and immunofluorescence analysis. BMSCs adhering to ASC scaffolds were implanted into the spinal cord hemisection cavities and were found to adhere well with native tissue and to form a bridge across the lesion cavity at 8 weeks after transplantation (Fig. 2). The effect of BMSCs seeded in ASC scaffolds on inflammatory cell regulation following SCI was evaluated by immunofluorescent staining for markers of inflammatory cells around the lesion sites at 2 weeks after surgery. CD68 and CD5 markers were used to evaluate the presence of macrophages and $\mathrm{T}$ lymphocytes, respectively. When BMSCs seeded in ASC scaffolds were implanted, the numbers of macrophages (microglia) and $\mathrm{T}$ lymphocytes $(\mathrm{P}<0.05)$ were significantly decreased around the SCIs when compared with the other groups at 2 weeks after surgery. However, the results of $\operatorname{IgM}$ staining revealed no significant differences in IgM-positive expression levels among the three groups ( $\mathrm{P}>0.05$; Figs. 3 and 4 ). These results indicate that BMSCs played a role in decreasing the number of inflammatory cells at the surgical site.

Analysis of cell apoptosis. Tissue specimens were analyzed by TUNEL assay to detect the localization of apoptotic cells by green fluorescence and of cell nuclei by blue fluorescence

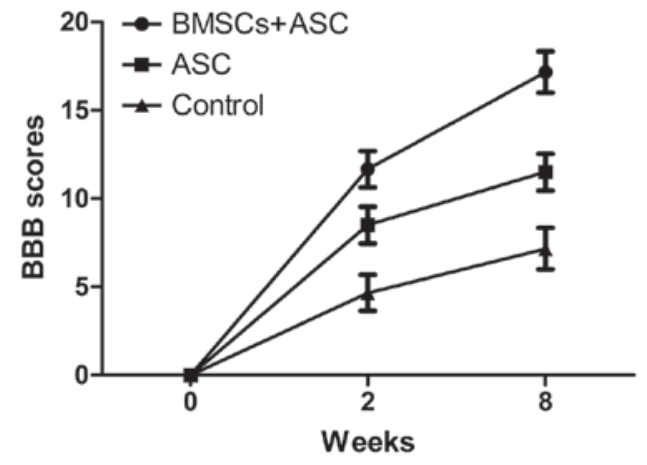

Figure 1. Behavioral assessment by BBB scoring after spinal cord injury $\mathrm{BBB}$, Basso, Beattie and Bresnahan; BMSCs, bone marrow stromal cells; ASC, acellular spinal cord.

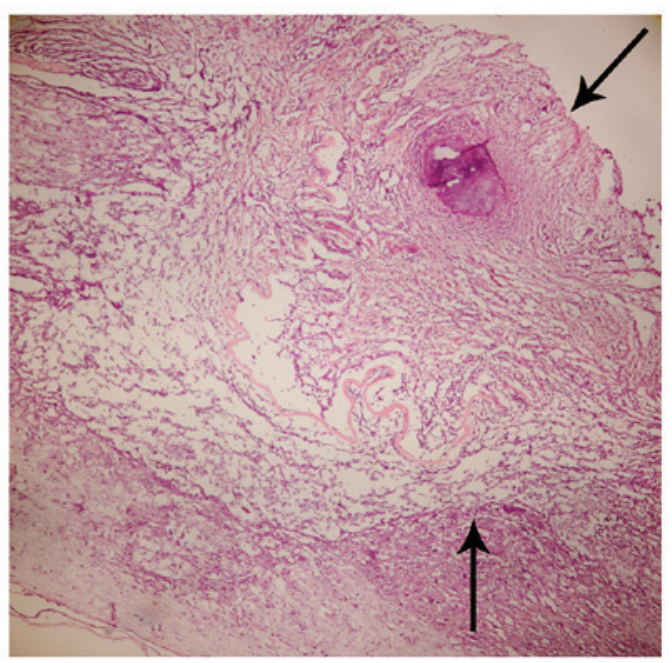

Figure 2. Coronal sections of hematoxylin and eosin staining of graft, comprising acellular spinal cord and bone marrow stromal cells, transplanted into the gap of a hemisected thoracic spinal cord 8 weeks after spinal cord injury. The graft (arrows indicate graft zones) integrated well with the host spinal cord.

using fluorescence microscopy. The extent of apoptosis among the BMSCs in the ASC scaffold group was significantly decreased, as compared with the other groups $(\mathrm{P}<0.05$; Fig. 5). However, there was no significant difference between the ASC group and the control group, indicating that the seeded BMSCs effectively resisted apoptosis.

\section{Discussion}

In the current study, ASC scaffolds seeded with BMSCs were implanted into spinal cord defects in hemisections of SD rats with SCI, as described in our previous study (8), to investigate the roles of BMSCs in the control of inflammation around grafts in the repair of SCIs. SCI causes the blood-spinal cord barrier to break down, which elicits an inflammatory response and progressive hemorrhagic necrosis and apoptosis at the lesion epicenter. As a result, catastrophic destruction of the structure of the spinal cord occurs with subsequent functional disability below the injury site (11). Accordingly, control of inflammation holds promise for the improvement of SCI repair (12). The results of the present 

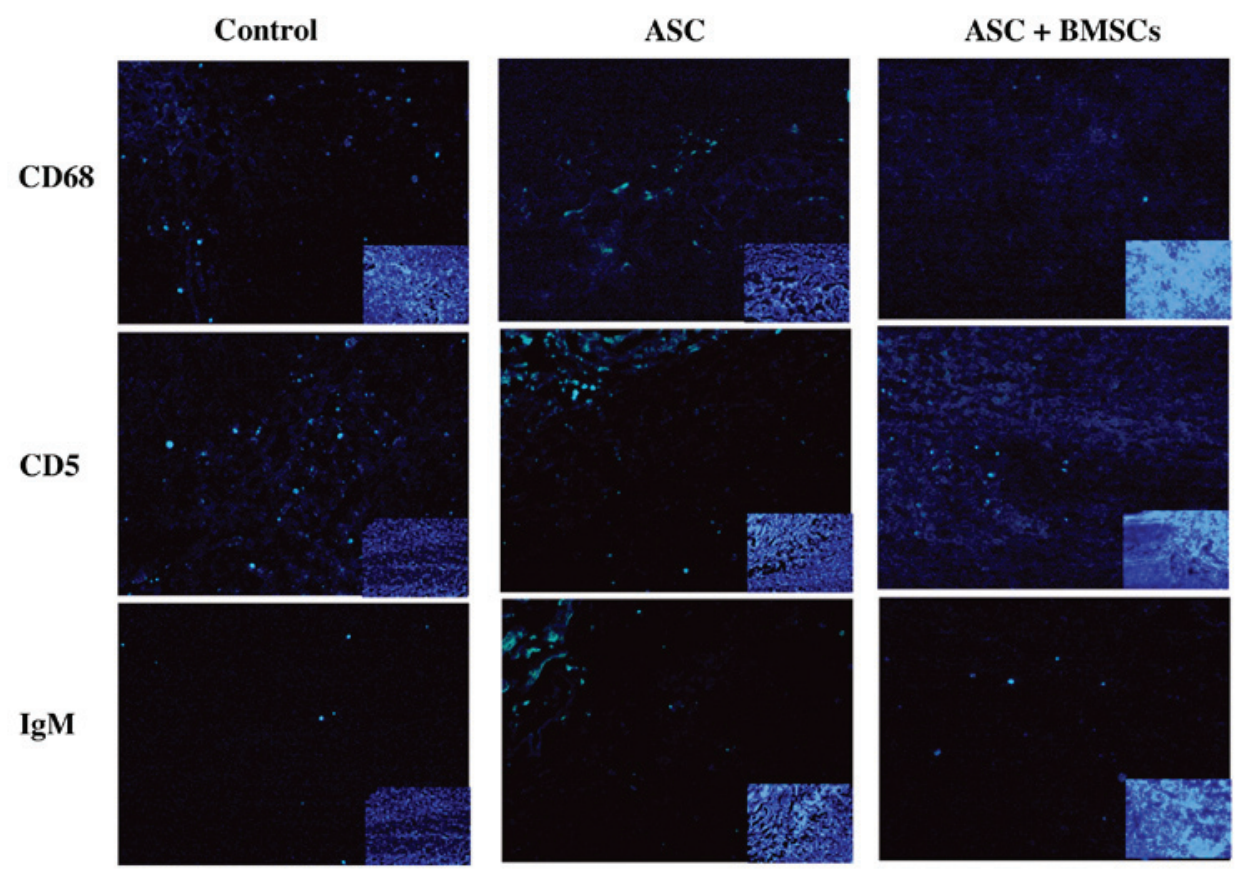

Figure 3. Inflammatory positive cells (green) and IgM (green) distribution at the lesion site 2 weeks after SCI (small inset to the lower right indicates blue 4',6-diamidino-2-phenylindole staining). Main image, magnification x400; inset image, magnification x40. SCI, spinal cord injury; ASC, acellular spinal cord; BMSCs, bone marrow stromal cells; CD, cluster of differentiation; IgM, immunoglobulin M.
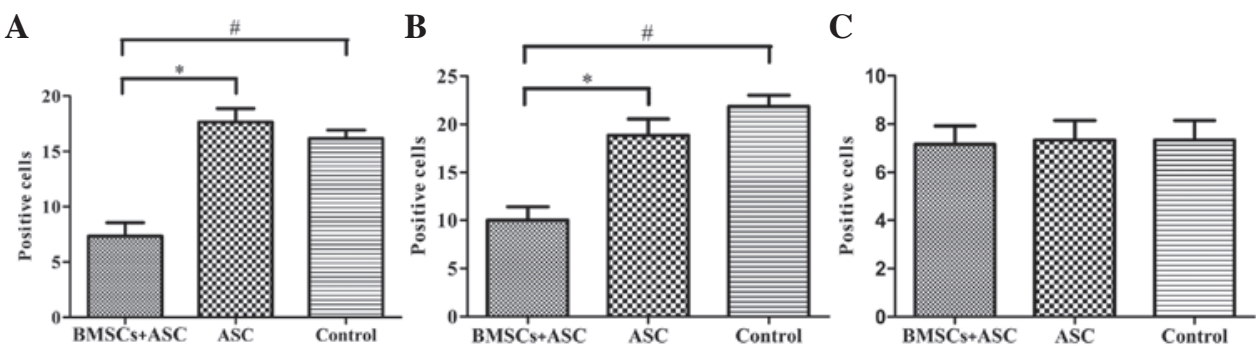

Figure 4. Quantitative comparison of the number of positive cells per field for (A) macrophages (CD68) and (B) T lymphocytes (CD5) showing significant differences for the BMSCs + ASC group compared with the ASC group $\left({ }^{*} \mathrm{P}<0.05\right)$ and control group $\left({ }^{*} \mathrm{P}<0.05\right)$ at 2 weeks after surgery. (C) IgM shows no significant difference $(\mathrm{P}>0.05)$ among the groups. ASC, acellular spinal cord; BMSCs, bone marrow stromal cells; IgM, immunoglobulin M.

A

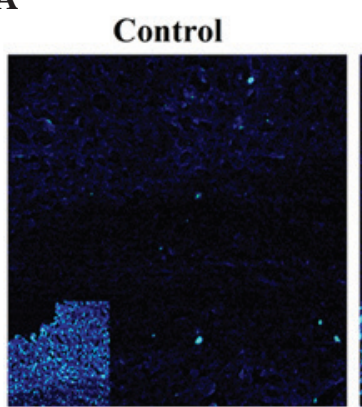

ASC

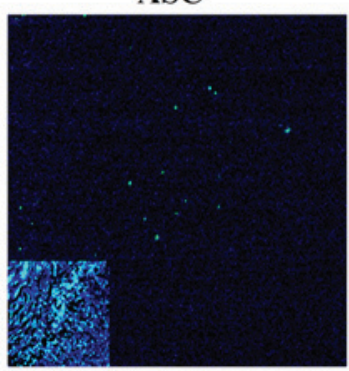

BMSCs-ASC

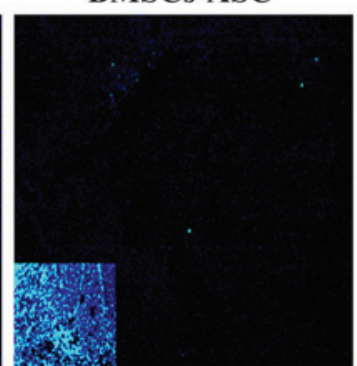

B

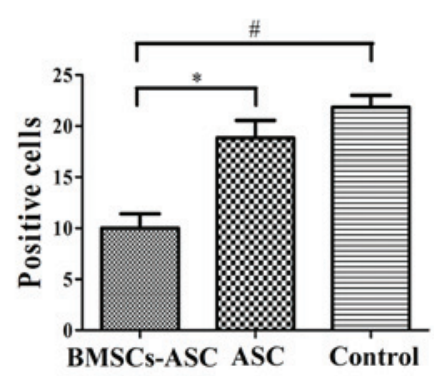

Figure 5. Distribution of TUNEL-positive cells around the injury sit (green, TUNEL; blue, DAPI). (A) Less positive TUNEL staining was visible at the injury sites of the ASC + BMSCs group. Main image, magnification x400; inset image, magnification x40. Insets indicate DAPI staining of contraction figure in main images. (B) Quantitative comparison of the positive cells shown by TUNEL among groups. Significantly fewer positively stained cells were observed in the BMSCs + ASC group at 2 weeks post-spinal cord injury compared with that in the other two groups ( $\left.{ }^{*} \mathrm{P}<0.05,{ }^{\sharp} \mathrm{P}<0.05\right)$. TUNEL, terminal deoxynucleotidyl transferase dUTP nick end labeling; DAPI, 4',6-diamidino-2-phenylindole; ASC, acellular spinal cord; BMSCs, bone marrow stromal cells.

study demonstrated that BMSCs seeded in ASC scaffolds are able to decrease the distribution of blood-derived immune cells around the SCI to promote functional recovery.
The results also indicated that ASC scaffolds seeded with BMSCs have good biocompatibility when used to bridge lesions in the host spinal cord. Moreover, the use of an ASC 
scaffold alone or with BMSCs significantly improved the recovery of locomotor function, in accordance with the results of our previous study (8). BBB scores also indicated that rats implanted with ASC scaffolds seeded with BMSCs exhibited better recovery of locomotor function than those treated with ASC scaffold alone or the untreated SCI group at 2 and 8 weeks after SCI. The use of BMSCs seeded in ASC scaffolds is a novel approach for the control of inflammation and reduction of tissue damage to promote functional recovery after SCI.

Markers of immune cells were used to evaluate the inflammatory response of the grafts implanted for SCI repair. Previous evidence suggests that leukocytes, particularly $\mathrm{T}$ lymphocytes and macrophages, which infiltrate the injured spinal cord are directly involved in the pathogenesis and extension of SCIs. Moreover, certain inflammatory processes, such as the production of cytokines, proteolytic enzymes and oxidative metabolites, exacerbate injury (13). In addition, inflammatory reactions can occur for weeks after SCI, with induction of the recruitment of macrophages and $\mathrm{T}$ cells from hours to weeks after injury $(14,15)$. The results of the present study demonstrated that the extent of inflammation caused by hematogenous macrophages and T lymphocytes in the SCI site was decreased by BMSCs seeded in scaffolds, suggesting that the BMSCs decreased the migration of inflammatory cells $(16,17)$. A possible explanation of this phenomenon is that BMSCs have low expression levels of histocompatibility complex antigens (class II) and release soluble factors and cytokines to regulate the inflammation reaction in response to SCI $(17,18)$. However, neither ASC alone or ASC combined with BMSCs showed a regulatory effect on IgM expression. On the basis of these observations, BMSCs appear to convey an advantage in cell-based therapy protocols, as transplantation improved the condition of the microenvironment and promoted motor function by reducing the recruitment of inflammatory cells early after trauma to improve SCI repair.

Apoptosis is a physiological process of programmed cell death that is essential for normal tissue development. Thus, resistance to apoptosis following SCI can directly benefit the repair of neural cells. In the present study, extensive cell apoptosis was observed by TUNEL assay in the control group and the group treated with ASC scaffold alone, but apoptosis was significantly decreased in rats implanted with ASC plus BMSCs. Hence, BMSCs play an important role by inhibiting the extent of apoptosis of cells in both the white and grey matter of the spinal cord. Although the cell types were not identified, it may be speculated that they are oligodendroglia cells, on the basis of our previous study (8).

In conclusion, the findings of this study demonstrated that BMSCs with ASC scaffolds significantly improve functional recovery through control of apoptosis and inflammation in the early recovery period following SCI trauma. Therefore, this model presents a potential therapeutic strategy for repair of SCIs. However, future studies are required to further elucidate the molecular mechanisms underlying the reestablishment of spinal cord function.

\section{Acknowledgements}

This study was supported by a grant from the Natural Science Foundation of Ningxia Province (grant no. NZ14288).

\section{References}

1. Rossi SL and Keirstead HS: Stem cells and spinal cord regeneration. Curr Opin Biotechnol 20: 552-562, 2009.

2. Enzmann GU, Benton RL, Talbott JF, Cao Q and Whittemore SR: Functional considerations of stem cell transplantation therapy for spinal cord repair. J Neurotrauma 23: 479-495, 2006.

3. Bartholdi D and Schwab ME: Methylprednisolone inhibits early inflammatory processes but not ischemic cell death after experimental spinal cord lesion in the rat. Brain Res 672: 177-186, 1995.

4. Beck KD, Nguyen HX, Galvan MD, Salazar DL, Woodruff TM and Anderson AJ: Quantitative analysis of cellular inflammation after traumatic spinal cord injury: Evidence for a multiphasic inflammatory response in the acute to chronic environment. Brain 133: 433-447, 2010.

5. Amar AP and Levy ML: Pathogenesis and pharmacological strategies for mitigating secondary damage in acute spinal cord injury. Neurosurgery 44: 1027-1039; discussion 1039-1040, 1999.

6. Esposito E, Mazzon E, Paterniti I, Impellizzeri D, Bramanti P and Cuzzocrea S: Olprinone attenuates the acute inflammatory response and apoptosis after spinal cord trauma in mice. PloS One 5: e12170, 2010.

7. Esposito E, Bruscoli S, Mazzon E, Paterniti I, Coppo M, Velardi E, Cuzzocrea S and Riccardi C: Glucocorticoid-induced leucine zipper (GILZ) over-expression in $\mathrm{T}$ lymphocytes inhibits inflammation and tissue damage in spinal cord injury. Neurotherapeutics 9: 210-225, 2012.

8. Chen J, Zhang Z, Liu J, Zhou R, Zheng X, Chen T, Wang L, Huang M, Yang C, Li Z, et al: Acellular spinal cord scaffold seeded with bone marrow stromal cells protects tissue and promotes functional recovery in spinal cord-injured rats. J Neurosci Res 92: 307-317, 2014.

9. Liu J, Chen J, Liu B, Yang C, Xie D, Zheng X, Xu S, Chen T, Wang L, Zhang Z, et al: Acellular spinal cord scaffold seeded with mesenchymal stem cells promotes long-distance axon regeneration and functional recovery in spinal cord injured rats. J Neurol Sci 325: 127-136, 2013.

10. Basso DM, Beattie MS and Bresnahan JC: A sensitive and reliable locomotor rating scale for open field testing in rats. J Neurotrauma 12: 1-21, 1995.

11. Bracchi-Ricard V, Lambertsen KL, Ricard J, Nathanson L, Karmally S, Johnstone J, Ellman DG, Frydel B, McTigue DM and Bethea JR: Inhibition of astroglial NF- $\mathrm{KB}$ enhances oligodendrogenesis following spinal cord injury. J Neuroinflammation 10: 92, 2013.

12. Wang CY, Chen JK, Wu YT, Tsai MJ, Shyue SK, Yang CS and Tzeng SF: Reduction in antioxidant enzyme expression and sustained inflammation enhance tissue damage in the subacute phase of spinal cord contusive injury. J Biomed Sci 18: 13, 2011.

13. de Rivero Vaccari JP, Lotocki G, Marcillo AE, Dietrich WD and Keane RW: A molecular platform in neurons regulates inflammation after spinal cord injury. J Neurosci 28: 3404-3414, 2008.

14. Bareyre FM and Schwab ME: Inflammation, degeneration and regeneration in the injured spinal cord: Insights from DNA microarrays. Trends Neurosci 26: 555-563, 2003.

15. Bethea JR and Dietrich WD: Targeting the host inflammatory response in traumatic spinal cord injury. Curr Opin Neurol 15: 355-360, 2002.

16. Lei J, Wang Z, Hui D, Yu W, Zhou D, Xia W, Chen C, Zhang Q and Xiang AP: Ligation of TLR2 and TLR4 on murine bone marrow-derived mesenchymal stem cells triggers differential effects on their immunosuppressive activity. Cell Immunol 271: 147-156, 2011.

17. Watanabe S, Uchida K, Nakajima H, Matsuo H, Sugita D, Yoshida A, Honjoh K, Johnson WE and Baba H: Early transplantation of mesenchymal stem cells after spinal cord injury relieves pain hypersensitivity through suppression of pain-related signaling cascades and reduced inflammatory cell recruitment. Stem Cells 33: 1902-1914, 2015.

18. Kim HJ, Park JB, Lee JK, Park EY, Park EA, Riew KD and Rhee SK: Transplanted xenogenic bone marrow stem cells survive and generate new bone formation in the posterolateral lumbar spine of non-immunosuppressed rabbits. Eur Spine J 17: 1515-1521, 2008 